\title{
LA FORMACIÓN ÉTICA DE LOS ESTUDIANTES DE MEDICINA: LA BRECHA ENTRE EL CURRÍCULO FORMAL Y EL CURRÍCULO OCULTO
}

\author{
Fernando Suárez Obando* y Eduardo Díaz Amado**
}

\begin{abstract}
Resumen: Tomar decisiones acertadas desde el punto de vista ético-moral en medicina es un asunto complejo y aprender a hacerlo debe constituir uno de los objetivos del entrenamiento médico. Una forma de contribuir a lograrlo es integrar una completa formación teórica en Ética Médica y Bioética con una verdadera correlación frente a lo vivido en la práctica clínica. Tener en cuenta lo que sienten y piensan los estudiantes al respecto es un buen referente para comenzar. En el presente artículo se presentan los resultados de un taller sobre el tema, llevado a cabo con estudiantes de medicina en una etapa avanzada de su formación, junto a las reflexiones suscitadas en el desarrollo de dicho taller.
\end{abstract}

Palabras clave: dilemas éticos, práctica clínica, currículo, educación médica

\section{ETHICS TRAINING OF MEDICAL STUDENTS: THE GAP BETWEEN THE FORMAL CURRICULUM AND THE HIDDEN CURRICULUM}

\begin{abstract}
To take right decisions in medicine from an ethical-moral point of view is a complex matter and to learn to do it must constitute one of the goals of medical training. A way to contribute to achieve this is to integrate a complete theoretical training in Medical Ethics and Bioethics with a true correlation in clinical practice experience. To take into account what students think and feel about the issue is a good reference to start. In the present paper the results on a workshop about the topic, carried out with medical students in advance training, are presented together with reflections raise in the workshop development.
\end{abstract}

Key words: ethical dilemmas, clinical practice, curriculum, medical education

\section{A FORMAÇÃO ÉTICA DOS ESTUDANTES DE MEDICINA: A DISTÂNCIA ENTRE O CURRÍCULO FORMAL E O CURRÍCULO OCULTO}

Resumo: Tomar decisões acertadas do ponto de vista ético-moral em Medicina é uma questão complexa e aprender a fazê-las constitui-se em um dos objetivos da formação médica. Uma forma de contribuir a esta questão é integrar uma ampla formação teórica de Ética Médica e Bioética relacionando-a à vivência na prática clínica. Levar em conta o que sentem e pensam os estudantes a respeito desta questão é um bom referencial inicial. O presente artigo apresenta os resultados de uma oficina sobre o tema, envolvendo os estudantes de Medicina em um estágio avançado de sua formação, além das reflexões advindas do desenvolvimento desta oficina.

Palavras chave: dilemas éticos, prática clínica, currículo, educação médica

* Médico genetista, profesor Instituto de Genética Humana, Pontificia Universidad Javeriana, Colombia

** Médico y filósofo, profesor Instituto de Bioética, Pontificia Universidad Javeriana, Colombia

Correspondencia: fernando.suarez@javeriana.edu.co 


\section{Introducción}

Los cursos de Ética Médica y Bioética (EMB) se encuentran integrados a los currículos de prácticamente todos los programas de medicina del país. Este hecho obedece a que la formación médica actual debe contemplar los diversos aspectos constitutivos de la complejidad que caracteriza a la profesión médica y que podríamos situar como "más allá de lo médico mismo". No es posible hablar de completa formación médica sin incluir en dicho proceso lo político, lo económico, lo jurídico $\mathrm{y}$, por supuesto, lo ético-moral, aspectos no meramente relacionados con la medicina sino fundamentalmente constitutivos de ella.

Desafortunadamente, y sólo para referirnos a la formación en EMB, la aproximación planteada en la mayoría de los casos está desvinculada de la realidad vivida en la práctica por los estudiantes. Lo frecuente es que no haya una constante integración entre ésta y los fundamentos teóricos que se dictan en las clases. Esta brecha puede generar, incluso, resultados opuestos a los deseados ${ }^{1}$. Se hace necesario, por tanto, realizar una aproximación práctica de la EMB que acompañe los imprescindibles fundamentos teóricos con una integración de las experiencias de los estudiantes de medicina dentro del desarrollo de los cursos de la facultad(1). Para comenzar esta integración es necesario realizar una aproximación descriptiva de la situación que el médico en entrenamiento

1 La discordancia entre teoría y práctica termina por llevar a los estudiantes a concluir que materias como Ética y Bioética son simples rellenos, que lo visto en ellas no tiene una verdadera utilidad en la práctica profesional y que ser médico es sólo un asunto "anatomofisiológico y farmacológico". Los resultados de esta situación se reflejan luego en una gran incapacidad de los médicos para tomar verdaderas decisiones prudentes (fundamentadas éticamente, lo cual incluye lo técnico y científico), para reconocer cuando hay un dilema ético en el transcurrir del ejercicio profesional, para construir mejores relaciones con sus pacientes y, finalmente, para actuar responsable y plenamente como sujetos morales y políticos, por ejemplo, frente a los demás colegas, a las instituciones o al Estado. enfrenta respecto de los dilemas éticos vividos en su práctica clínica.

Durante el curso de Bioética Clínica en la carrera de Medicina de la Universidad Javeriana se decidió realizar un taller que describiera la situación personal de los alumnos frente a los dilemas que enfrentaban en su práctica clínica. Como resultado de esta experiencia, se comprobó la existencia de una enorme brecha entre los planteamientos teóricos y la evidencia práctica.

\section{Materiales y métodos}

Los estudiantes de IX semestre de la Facultad de Medicina que cursaban la clase de Bioética Clínica (2006, primer semestre), en grupos de cinco personas, debían discutir y responder las siguientes preguntas:

- ¿Cree usted que la ética enseñada en la carrera de Medicina tiene un asidero real en la práctica clínica?

- ¿Considera que se deben implementar prácticas de ética médica durante las rotaciones clínicas?

- Enumere algunos dilemas éticos que ha enfrentado durante la práctica clínica y responda:

* ¿Cómo se han dilucidado estos dilemas?

* ¿Sabe el desenlace final de esos dilemas?

* ¿Quién o quiénes resolvieron los dilemas?

- Si usted como estudiante enfrenta un dilema ético durante la práctica clínica, ¿a quién recurre para resolverlo?

Las respuestas debían ser sostenidas mediante argumentos y los estudiantes tenían que haber leído previamente el artículo titulado: 
"Understanding the clinical dilemmas that shape medical students' ethical development: questionnaire survey and focus group study"(2)

El taller fue desarrollado por la totalidad de estudiantes del curso (30) en el plazo de una semana. Posteriormente, se revisaron todas las respuestas y se extrajo de cada una la idea más relevante, según se reflejara en la práctica clínica y en los temas desarrollados durante el curso de Bioética Clínica. Algunas se integraron bajo una sola, en los casos en que el argumento o la contestación fueran similares en diferentes grupos.

Este taller se realizó a mitad de semestre académico, es decir, cuando los estudiantes ya habían recibido las nociones fundamentales de Bioética Clínica. Además, se trató de estudiantes que ya habían tenido experiencia clínica, al menos desde el quinto semestre de la carrera, habiendo rotado por Medicina Interna, Psiquiatría, Cirugía, Ginecología y Obstetricia, Especialidades Quirúrgicas, Urgencias y Pediatría.

\section{Resultados}

Los grupos de estudiantes identificaron diversas características y situaciones que no necesariamente se contemplan, debaten o discuten durante el desarrollo de un currículo clásico de Ética Médica. De acuerdo con las respuestas se presentan los aspectos más relevantes en las tablas 1,2 y 3 .

Tabla 1

\begin{tabular}{|l|l|l|}
\hline Pregunta & Respuestas & Ejemplos y comentarios \\
\hline $\begin{array}{l}\text { ¿Cree usted que la ética } \\
\text { enseñada en la carrera } \\
\text { de Medicina tiene un } \\
\text { asidero real en la prác- } \\
\text { tica clínica? }\end{array}$ & $\begin{array}{l}\text { Sí. De manera independiente a la enseñanza de la ética médica en } \\
\text { la carrera, es la práctica clínica el asidero real de la ética. } \\
\text { laboral de los médicos, sino que esta es la que hace a los buenos } \\
\text { médicos. }\end{array}$ & \\
\hline $\begin{array}{l}\text { ¿Considera que se de- } \\
\text { ben implementar prác- } \\
\text { ticas de ética médica } \\
\text { durante las rotaciones } \\
\text { clínicas? }\end{array}$ & $\begin{array}{l}\text { Sí, las prácticas clínicas de ética médica deberían ser considera- } \\
\text { das en paralelo a las rotaciones clínicas. }\end{array}$ & $\begin{array}{l}\text { La rotación de ética clínica es la forma } \\
\text { como se definen en la práctica herra- } \\
\text { mientas útiles para tomar decisiones } \\
\text { acertadas en momentos críticos. }\end{array}$ \\
\hline
\end{tabular}

Respecto de la primera pregunta, los estudiantes afirman la importancia que tiene la ética en el desarrollo del médico y la experiencia derivada de la práctica clínica; respecto de la segunda, resaltan que, de manera paralela a las rotaciones clínicas, debe haber por lo menos tutores preparados para responder a preguntas éticas surgidas de la experiencia clínica.

Los alumnos describen situaciones clínicas vividas en la práctica y que incluyen elementos de relevancia ética. Los dilemas éticos derivados de esas vivencias generan interrogantes que, en la mayoría de los casos, no son resueltos ni discutidos con los estudiantes. Es decir, aunque los estudiantes puedan observar el procedimiento llevado a cabo o el resultado final que se obtuvo con determinado paciente, las conductas de los diferentes miembros del equipo de sa$\operatorname{lud}^{2}$ y los aspectos éticos que se derivan de los casos analizados no son expuestos claramente $\mathrm{o}$ incluidos formalmente dentro del proceso de formación de los estudiantes.

2 Aquí está incluido personal docente, personal de enfermería, residentes y personal administrativo. 
Tabla 2

\begin{tabular}{|c|c|c|}
\hline Pregunta & Casos, soluciones y dilemas & Ejemplos y comentarios \\
\hline \multirow[t]{4}{*}{$\begin{array}{l}\text { Enumere algunos dilemas éticos } \\
\text { que ha enfrentado durante la } \\
\text { práctica clínica y responda: } \\
\text { ¿Cómo se han dilucidado estos } \\
\text { dilemas? } \\
\text { ¿Sabe el desenlace final de esos } \\
\text { dilemas? } \\
\text { ¿Quién o quiénes resolvieron los } \\
\text { dilemas? }\end{array}$} & Resolución del caso & $\begin{array}{l}\text { Paciente neonato con múltiples malformaciones y pobre } \\
\text { pronóstico (Asociación VACTERL). } \\
\text { Equipo interdisciplinario del Hospital. La paciente fallece } \\
\text { después de múltiples intervenciones. } \\
\text { Dificultad en la toma de decisiones ante múltiples malfor- } \\
\text { maciones y definición de pronóstico, lo que perjudicó la } \\
\text { calidad de vida del afectado. }\end{array}$ \\
\hline & $\begin{array}{l}\text { Caso reanimación } \\
\text { Resolución del caso } \\
\text { Dilema identificado }\end{array}$ & $\begin{array}{l}\text { Estudiantes de Medicina encargados del manejo del AMBU } \\
\text { por más de } 10 \text { horas, en el cambio de turno no hay quién } \\
\text { reciba al paciente. } \\
\text { El paciente fallece. } \\
\text { Responsabilidades que exceden las capacidades del es- } \\
\text { tudiante. }\end{array}$ \\
\hline & $\begin{array}{l}\text { Caso cirugía } \\
\text { Resolución del caso } \\
\text { Dilema identificado }\end{array}$ & $\begin{array}{l}\text { Anciano con retardo mental y sin soporte social ni eco- } \\
\text { nómico que es sometido a cirugía vascular, con el fin de } \\
\text { enseñar a residentes e internos la técnica quirúrgica, sin } \\
\text { consentimiento, ni indicación quirúrgica. } \\
\text { No existió ninguna resolución o discusión debido a que ni } \\
\text { siquiera se planteó como un problema ético por parte del } \\
\text { equipo tratante. } \\
\text { Conflicto entre educación y cuidado del paciente, ausencia } \\
\text { de consentimiento informado y falta total de identificación } \\
\text { del caso como una conducta errada y antiética por parte } \\
\text { de los docentes. }\end{array}$ \\
\hline & $\begin{array}{l}\text { Caso reumatología } \\
\text { Resolución del caso } \\
\text { Dilema identificado }\end{array}$ & $\begin{array}{l}\text { Paciente con enfermedad reumatológica en quien se inicia } \\
\text { tratamiento con medicamentos potencialmente teratogéni- } \\
\text { cos, sin antes haber descartado embarazo. } \\
\text { No se ha resuelto hasta el momento. Paciente embarazada, } \\
\text { se desconocen efectos en el feto. } \\
\text { Atención médica percibida como irresponsable y de mala } \\
\text { calidad. }\end{array}$ \\
\hline
\end{tabular}

Se sitúa a la EMB no como una simple cátedra del currículo, sino como un componente fundamental en la formación y definición de un buen médico, y se enfatiza en que su desarrollo debe estar directamente relacionado con la experiencia del estudiante.

\section{Una rotación de Bioética Clínica o la presen-} cia de un tutor cualificado en este campo durante la práctica clínica se destacan en las respuestas de los estudiantes como propuestas que podrían contribuir a la resolución práctica de los dilemas. De esta manera, se contaría con un referente directo y cercano para discutir y resolver las dudas que se presenten, sin tener que esperar los resultados de un Comité Bioético Hospitalario, pocas veces disponible para los alumnos. Asimismo, se reduciría la discusión y resolución de dilemas vía "olfato moral", en la que éstos terminan siendo tratados más como un asunto de "intercambio libre de opiniones" que como un problema real al interior de la práctica clínica. 


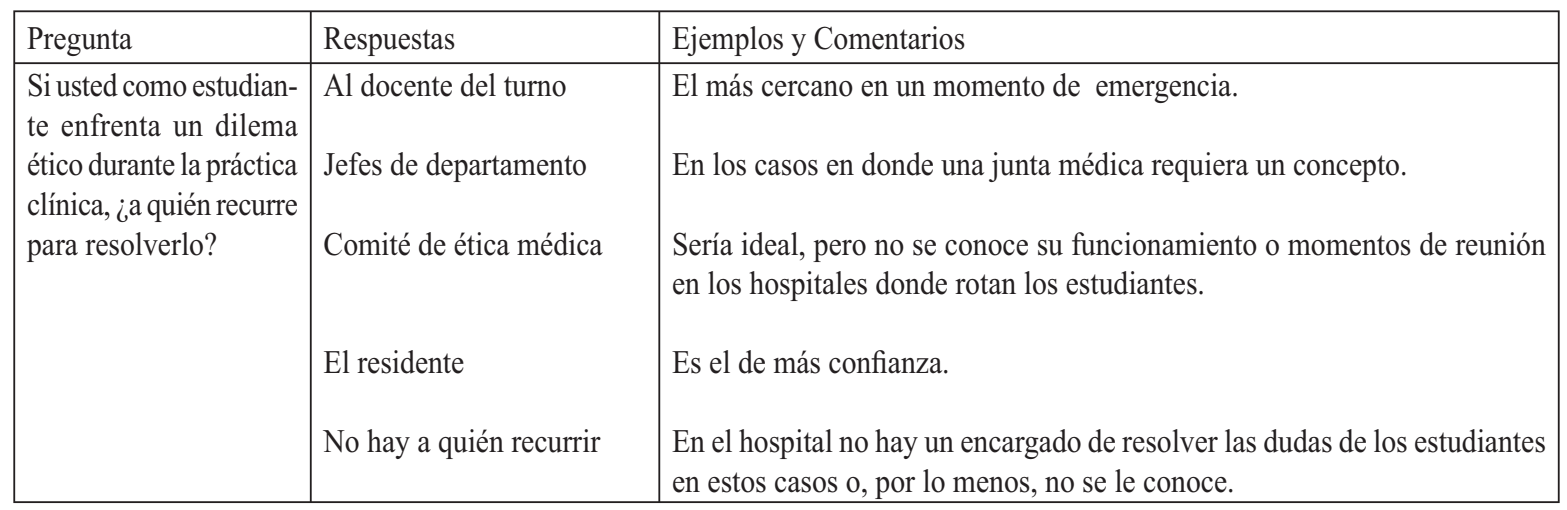

Los estudiantes identifican a algunas personas a quienes recurrir en caso de duda o dilema; sin embargo, éstas no siempre son las más preparadas y los comités, que deberían ser los indicados como punto de referencia, son desconocidos en su funcionamiento por parte de los alumnos.

A través de los casos descritos, se pueden identificar los siguientes aspectos relacionados con la formación ética de los estudiantes de medicina:

- Dificultad en la toma de decisiones en momentos críticos (caso pediatría).

- Responsabilidades que exceden las capacidades del estudiante (caso reanimación).

- Conflicto entre educación y cuidado del paciente con incapacidad y ausencia de consentimiento informado (caso cirugía).

- Atención médica percibida como irresponsable y de mala calidad (caso reumatología).

En la última pregunta se identifica a las personas a quienes se puede recurrir en caso de duda sobre dilemas éticos, incluyendo el residente, el docente y el comité de ética, a pesar de que, aunque se sabe de la existencia de este último, no se conoce el modo de acceder a él. Por último, se destaca la afirmación de que no hay un referente válido para los estudiantes que resuelva o escuche sus inquietudes en este sentido.

\section{Discusión}

Cabe anotar que la presente experiencia surge de las opiniones de un reducido número de estudiantes de la Carrera de Medicina y que no corresponde a una investigación formal que arroje resultados producto de un protocolo previamente diseñado para conocer las experiencias de los estudiantes. Sin embargo, constituye un indicador de lo que puede estar ocurriendo con el aprendizaje e interiorización de conceptos éticos, la capacidad para enunciar y analizar dilemas de este tipo en la práctica clínica y la adquisición de habilidades para su resolución en medio de la vivencia clínica durante la formación médica.

Las investigaciones que describen las situaciones vividas por los estudiantes tienen múltiples antecedentes en la literatura mundial, tales como los estudios de comprensión de los dilemas que influyen en la formación del médico y su resolución en los ámbitos clínicos(2), en los cuales los estudiantes describen su realidad clínica y el modo en que los dilemas éticos no son definidos como tales en la mayoría de los casos; por lo tanto, su resolución no es compartida por el cuerpo docente. También se han llevado a cabo investigaciones que analizan los conflictos éticos del interno o residente 
en relación con el ámbito cultural propio y el de sus pacientes(3), y se concluye que las facultades deben encontrar nuevas estrategias pedagógicas para apropiarse de esos modelos y lineamientos que, a su vez, sean puestos en práctica de acuerdo con lo que se espera de sus propios graduandos (futuros médicos).

La introducción de los dilemas éticos del estudiante en la propia enseñanza de la EMB se justifica a la luz de la experiencia personal del médico en entrenamiento y su correlación con los fundamentos teóricos generados en la cátedra o los seminarios de la Facultad(4).

Se requiere de una base empírica para el buen desarrollo de la cátedra de EMB en Medicina y ella sólo puede ser obtenida con la participación directa del estudiantado que realiza la práctica clínica(5). Obviamente, para que esto pueda darse se requiere también de la disposición adecuada de las diversas instancias de la Facultad y de los sitios donde se llevan a cabo las rotaciones.

Por un lado, el currículo formal propuesto en el pensum de la carrera se desdibuja en la práctica y, por otro, se desatiende la enorme influencia del "currículo oculto", que ejerce su influencia mediante el ejemplo que los profesores dan a sus alumnos, a través del ethos institucional que moldea los discursos (los modos de ver y de comunicar) y, también, mediante la adquisición de costumbres y rituales asumidos como normales $\mathrm{o}$, incluso, deseables(6). Sólo asumiendo abierta $\mathrm{y}$ frontalmente un verdadero proceso de formación ética es posible responder adecuadamente a los retos que plantea la formación de buenos médicos para el mundo contemporáneo, con buena enseñanza, buen ejemplo y promoción de conductas adecuadas(7).

Para el caso del taller realizado con los estudiantes y presentado en este artículo, esa influencia puede rastrearse a través de tres grandes aspectos: el primero, en el cual el ejemplo dado por los encargados de llevar a cabo un tratamiento se constituye en la conducta frecuente (se realiza un procedimiento quirúrgico teniendo en cuenta sólo el objetivo estratégico de hacer docencia, sin abordar interrogantes tales como: ¿de qué modo podría combinarse el aspecto estratégico con aspectos sustanciales, tales como el respeto debido a las personas, la limitación terapéutica o la justicia sanitaria?); el segundo, en el que las decisiones son tomadas heterónomamente ${ }^{3}$, sin discutir los modos y consecuencias de este tipo de decisiones: los comités resuelven, aunque no se sepa quiénes lo componen, cómo funcionan y qué responsabilidades -éticas, políticas y jurídicas- tienen dichos órganos y cada uno de sus integrantes; y un tercer escenario, en el cual se pone al descubierto que no hay referente claro y confiable a quien consultar desde la posición del estudiante.

\section{Conclusiones}

El presente artículo describe las opiniones de un grupo de estudiantes en el terreno de la EMB en relación con su vivencia en la clínica y permite realizar un primer acercamiento a la situación del médico en entrenamiento.

Permite observar que hay una discrepancia entre lo enseñado y lo vivido, y que para el estudiante no existe un referente concreto en el cual apoyarse, cuando existen dilemas o dudas sobre aspectos éticos de la práctica clínica.

Esta primera aproximación justifica el planteamiento de una investigación profunda sobre el tema y deja abierta la posibilidad de

3 Se habla de heteronomía, por oposición a autonomía, en situaciones en las cuales un "otro" es el que toma las decisiones (parientes, comité de bioética, personal médico, etc.), y no el directamente implicado por la decisión que se tome, en este caso el paciente. 
que los dilemas y vivencias de los estudiantes se integren a la clase de Bioética Clínica, con el fin de disminuir la influencia ejercida por el currículo oculto.
Finalmente, la investigación descriptiva, en razón de la participación del estudiante de Medicina, es un imperativo que se justifica a la luz de una ética fundamentada (teoría) y práctica (aplicada).

\section{Referencias}

1. Caldicott CV, Faber-Langendoen K. Deception, discrimination, and fear of reprisal: lessons in ethics from third-year medical students. Acad Med 2005; 80(9): 866-873.

2. Hicks LK, Lin Y, Robertson DW, Robinson DL, Woodrow SI. Understanding the clinical dilemmas that shape medical students' ethical development: questionnaire survey and focus group study. BMJ 2001; 322(7288): 709-710.

3. Notzer N, Abramovitch H, Dado-harari R, et al. Medical students' ethical, legal and cross-cultural experiences during their clinical studies. Isr Med Assoc J 2005; 7(1): 58-61.

4. St Onge J. Medical education must make room for student-specific ethical dilemmas. CMAJ 1997; 156(8): 1175-1177.

5. Huijer M, van Leeuwen E, Boenink A, et al. Medical students' cases as an empirical basis for teaching clinical ethics. Acad Med 2000; 75(8): 834-9.

6. Restrepo LC, Espinel M. Semiología de las prácticas de salud. Bogotá: CEJA; 1996.

7. Boon K, Turner J. Ethical and professional conduct of medical students: review of current assessment measures and controversies. $J$ Med Ethics 2004; 30: 221-226.

Recibido el 11 de julio de 2006.

Aceptado el 13 de marzo de 2007. 